\title{
ON A MULTIDIMENSIONAL VOLKENBORN INTEGRAL AND HIGHER ORDER BERNOULLI NUMBERS
}

\author{
MIN-SOO KIM AND Jin-WOO SON
}

In this paper, using a multidimensional Volkenborn integral, we give a $p$-adic expression of the higher order Bernoulli numbers. This shows immediately the relation to the sums of products of the ordinary Bernoulli numbers of Dilcher in 1996. We also consider the Mahler expansion of several $p$-adic variables function, and give some examples.

\section{INTRODUCTION AND NOTATION}

The Bernoulli polynomials of order $k$, denoted $B_{n}^{(k)}(x)$, are defined by

$$
\left(\frac{t}{e^{t}-1}\right)^{k} e^{x t}=\sum_{n=0}^{\infty} B_{n}^{(k)}(x) \frac{t^{n}}{n !} .
$$

In particular, the values at $x=0$ are called Bernoulli numbers of order $k$, that is, $B_{n}^{(k)}(0)=B_{n}^{(k)}($ see $[1,2,4,5,9,10,14])$. When $k=1$, the polynomials or numbers are called ordinary.

The polynomials $B_{n}^{(k)}(x)$ and numbers $B_{n}^{(k)}$ were first defined and studied by Nörlund [9]. Also Carlitz [2] and others investigated their properties. Recently they have been studied by Adelberg [1], Howard [5], and Young [14]. In [1], Adelberg has given congruences for $B_{n}^{(k)}$ which extended the Kummer congruences and has deduced information concerning the irreducibility of certain Bernoulli polynomials with order divisible by $p$. Howard [5] investigated other numbers related to the higher order Bernoulli numbers. Young [14] considered the $p$-adic integrals and measures to obtain congruences for the higher order Bernoulli numbers and polynomials.

In this paper, using a multidimensional Volkenborn integral, we give a $p$-adic expression for Bernoulli number of order $k$. As an easy corollary we see that a $p$-adic expression for the higher order Bernoulli number is related to the sums of products of the ordinary Bernoulli numbers in Dilcher [4]. We give some examples. Our approach essentially coincides with the $p$-adic expression for the ordinary Bernoulli numbers

Received 26th April. 2001

Copyright Clearance Centre, Inc. Serial-fee code: 0004-9727/02 \$A2.00+0.00. 
introduced by many authors, see for example, $[8, \mathbf{1 1}, \mathbf{1 2}]$. We also consider the Mahler expansion of several $p$-adic variables function and its examples.

We denote by $p$ an arbitrary but fixed rational prime number throughout. We shall use $\mathbb{N}, \mathbb{Z}, \mathbb{Z}_{p}, \mathbb{Q}_{p}$ and $\mathbb{C}_{p}$ for, respectively, the set of natural numbers, the ring of rational integer, the ring of $p$-adic integer, the field of $p$-adic numbers, and the completion of the algebraic closure of $\mathbb{Q}_{p} . B_{n}$ will be the $n$th ordinary Bernoulli number defined by the generating function $t /\left(e^{t}-1\right)$. For $s \in \mathbb{C}_{p}$ write $\operatorname{ord}_{p}(s)$ for the $p$-adic ordinal of $s$. It is easy to check ord $\mathrm{d}_{p}$ satisfies the following three properties.

(a) $\operatorname{ord}_{p}(s t)=\operatorname{ord}_{p}(s)+\operatorname{ord}_{p}(t)$;

(b) $\operatorname{ord}_{p}(s+t) \geq \min \left\{\operatorname{ord}_{p}(s), \operatorname{ord}_{p}(t)\right\}$;

(c) $\operatorname{ord}_{p}(s)=\infty$ if and only if $s=0$.

\section{Some Results}

Let $d$ be a fixed positive integer. We set $X_{d}=\lim _{N}\left(\mathbb{Z} / d p^{N} \mathbb{Z}\right)$, the map from $\mathbb{Z} / d p^{M} \mathbb{Z}$ to $\mathbb{Z} / d p^{N} \mathbb{Z}$ for $M \geq N$, to be reduction $\bmod d p^{N}$. In the special case $d=1$, $X_{1}=\mathbb{Z}_{p}$. Let $a+p^{N} \mathbb{Z}_{p}=\left\{x \in \mathbb{Q}_{p}|| x-\left.a\right|_{p} \leq p^{-N}\right\}$ for $a \in \mathbb{Q}_{p}$ and $N \in \mathbb{Z}$. Then the sets of the form $a+p^{N} \mathbb{Z}_{p}$ form a basis of open sets for the metric space $\mathbb{Q}_{p}$. This means that any open subset of $\mathbb{Q}_{p}$ is a union of open subsets of this type. Note that (see $[6,7,11])$

$$
a+d p^{N} \mathbb{Z}_{p}=\bigcup_{0 \leq b<p}\left(a+b d p^{N}\right)+d p^{N+1} \mathbb{Z}_{p}, \quad X_{d} \backslash p X_{d}=\bigcup_{\substack{0<a<d p \\(a, p)=1}} a+d p \mathbb{Z}_{p} .
$$

Let $U D\left(\mathbb{Z}_{p}, \mathbb{C}_{p}\right)$ be the Banach algebra of all uniformly (or strictly) differentiable functions $f: \mathbb{Z}_{p} \rightarrow \mathbb{C}_{p}$ under the pointwise operations and valuation (see $[\mathbf{1 0}, \mathbf{1 1}, \mathbf{1 3}]$ ).

Let $\mu_{0}\left(a+d p^{N} \mathbb{Z}_{p}\right)=1 /\left(d p^{N}\right)$. The Volkenborn integral of a function $f \in$ $U D\left(\mathbb{Z}_{p}, \mathbb{C}_{p}\right)$ is defined by (see $[\mathbf{1 0}, \mathbf{1 1}]$ )

(2) $\int_{X_{d}} f(x) d \mu_{0}(x):=\lim _{N \rightarrow \infty} \sum_{a=0}^{d p^{N}-1} f(a) \mu_{0}\left(a+d p^{N} \mathbb{Z}_{p}\right)=\lim _{N \rightarrow \infty}\left(1 / d p^{N}\right) \sum_{a=0}^{d p^{N}-1} f(a)$

using the $p$-adic limit of the $N$ th Riemann sum of $f$. Here are the main properties of this integral.

Proposition 1. (See $[8,9,11,12]$ ) Let $|t|_{p} \leq p^{1 /(1-p)}, t \in \mathbb{C}_{p}$ and $t \neq 0$, and let $\chi$ be a primitive Dirichlet character with conductor $d$. Then we have
1. $\int_{\mathbb{Z}_{p}} e^{t x} d \mu_{0}(x)=t /\left(e^{t}-1\right)$
2. $B_{n}=\int_{\mathbb{Z}_{p}} x^{n} d \mu_{0}(x)$. 
3. $\int_{X_{d}} \chi(x) e^{t x} d \mu_{0}(x)=\sum_{a=0}^{d-1}\left(\chi(a) t e^{a t}\right) /\left(e^{d t}-1\right)$.

4. $B_{n, \chi}=\int_{X_{d}} x^{n} \chi(x) d \mu_{0}(x)$, where $B_{n, \chi}$ are the generalised ordinary Bernoulli numbers, that is, $B_{n, \chi} / n$ ! is the coefficient of $t^{n}$ in $\sum_{a=0}^{d-1}\left(\chi(a) t e^{a t}\right) /\left(e^{d t}-1\right)$.

Note that Part 2 and Part 4 of Proposition 1 are called the $p$-adic expression for the ordinary Bernoulli numbers and the generalised ordinary Bernoulli numbers, respectively.

For a fixed $k \in \mathbb{N}$, we set $\mathbf{X}=\prod_{\substack{d_{i} \in \mathbb{N} \\ i=1, \ldots, k}} X_{d_{i}}$ with the product topology, so $\mathbf{X}$ is compact since $X_{d_{i}}$ is compact for $i=1, \ldots, k$.

The notations $\mathbf{a}$ and $\mathbf{x}$ denote, respectively, $\left(a_{1}, \ldots, a_{k}\right)$ and $\left(x_{1}, \ldots, x_{k}\right)$.

The vector space $\mathbb{Q}_{p}^{k}$ has the norm $\|\mathbf{x}\|_{p}=\max _{1 \leq i \leq k}\left|x_{i}\right|_{p}$ and satisfies the nonArchimedean property $\|\mathbf{x}+\mathbf{y}\|_{p} \leq \max \left(\|\mathbf{x}\|_{p},\|\mathbf{y}\|_{p}\right)$ for $\mathbf{x}, \mathbf{y} \in \mathbb{Q}_{p}^{k}$. Let $\mathbf{d}$ be a point in $\mathbb{N}^{k}$. We shall consider the polydisc

$$
\mathbf{a}+\mathbf{d} p^{\mathbf{N}} \mathbb{Z}_{p}=\left\{\mathrm{x} \in \mathbb{Q}_{p}^{k} \mid x_{i} \equiv a_{i}\left(\bmod d_{i} p^{N_{i}}\right), i=1, \ldots, k\right\}
$$

where $\mathbf{a} \in \mathbb{Q}_{p}^{k}$ and $\mathbf{d} p^{\mathbf{N}}=\left(d_{1} p^{N_{1}}, \ldots, d_{k} p^{N_{k}}\right)$ for $\mathbf{N} \in \mathbb{N}^{k}:$ that is, the point $\mathbf{a}$ is the least nonnegative residue of $\mathbf{x} \bmod \mathbf{d} p^{\mathbf{N}}$. The polydisc $\mathbf{a}+\mathbf{d} p^{N} \mathbb{Z}_{p}$ is the product of $\operatorname{discs} a_{i}+d_{i} p^{N_{i}} \mathbb{Z}_{p}$ for $i=1, \ldots, k$,

$$
\mathbf{a}+\mathbf{d} p^{\mathbf{N}} \mathbb{Z}_{p}=\left(a_{1}+d_{1} p^{N_{1}} \mathbb{Z}_{p}\right) \times \cdots \times\left(a_{k}+d_{k} p^{N_{k}} \mathbb{Z}_{p}\right) .
$$

Definition 1: Let $\mathbf{a}+\mathbf{d} p^{\mathbf{N}} \mathbb{Z}_{p}$ be a polydisc with $\mathbf{a} \in \mathbb{Q}_{p}^{k}$ and $\mathbf{d}=\left(d_{i}\right)_{1 \leq i \leq k} \in \mathbb{N}^{k}$. Let $\mu_{i}$ be a distribution on $\mathbb{Z}_{p}$ for $i=1,2, \ldots, k$. We define a formal direct product of distributions $\mu(\mathbf{a})=\mu\left(\mathrm{a}+\mathrm{d} p^{N} \mathbb{Z}_{p}\right)$ by

$$
\mu\left(\mathbf{a}+\mathbf{d} p^{\mathbb{N}} \mathbb{Z}_{p}\right):=\mu_{1}\left(a_{1}\right) \ldots \mu_{k}\left(a_{k}\right):=\mu_{1}\left(a_{1}+d_{1} p^{N_{1}} \mathbb{Z}_{p}\right) \ldots \mu_{k}\left(a_{k}+d_{k} p^{N_{k}} \mathbb{Z}_{p}\right) .
$$

Definition 2: $([3,8]) \quad$ We call a function defined on a subset of $\mathbb{C}_{p}^{k}$ holomorphic if it can be represented by a single power series and locally holomorphic if at each point in the domain we can represent the function by a power series on some polydisc containing the point.

Let $\mathcal{O}$ be an open set in $\mathbb{C}_{p}^{k}$ with $\mathbf{a}+\mathbf{d} p^{\mathbf{N}} \mathbb{Z}_{p} \subset \mathbf{X} \subset \mathcal{O}$. Suppose $\mathcal{B}$ is a Banach space over $\mathbb{C}_{p}$ and $f: \mathcal{O} \rightarrow \mathcal{B}$ is locally holomorphic. For $\left(N_{1}, \ldots, N_{k}\right) \in \mathbb{N}^{k}$, we say 
that $\left\{N_{i}\right\} \rightarrow \infty$ when $N_{1} \rightarrow \infty, \ldots, N_{k} \rightarrow \infty$. Define

$$
\begin{aligned}
& \int_{\mathbf{X}} f(\mathbf{x}) d \mu(\mathbf{x}):=\int_{X_{d_{1}}} \ldots \int_{X_{d_{k}}} f\left(x_{1}, \ldots, x_{n}\right) d \mu_{1}\left(x_{1}\right) \ldots d \mu_{k}\left(x_{k}\right) \\
&=\lim _{\left\{N_{i}\right\} \rightarrow \infty} \sum_{a_{1}=0}^{d_{1} p^{N_{1}-1}} \ldots \sum_{a_{k}=0}^{d_{k} p^{N_{k}-1}} f(\mathbf{a}) \mu\left(\mathbf{a}+\mathbf{d} p^{\mathbf{N}} \mathbb{Z}_{p}\right)
\end{aligned}
$$

where $\mathbf{a}=\left(a_{1}, \ldots, a_{k}\right)$ and $\mathbf{d}=\left(d_{1}, \ldots, d_{k}\right) \in \mathbb{N}^{k}$ (see [3, Theorem 1]).

A multi-index $\mathbf{J}$ is an element of the $k$-tuples of nonnegative integers. Let $\mathbf{J}=$ $\left(j_{1}, \ldots, j_{k}\right)$ be multi-index and $\mathbf{x}=\left(x_{1}, \ldots, x_{k}\right) \in \mathcal{O}$. We use the abbreviations, $\mathbf{x}^{\mathbf{J}}=x_{1}^{j_{1}} \ldots x_{k}^{j_{k}}$ and $|\mathbf{J}|=j_{1}+\cdots+j_{k}$.

Suppose that $f(\mathbf{x})$ is given by a power series

$$
f(\mathbf{x})=\sum_{\mathbf{J}} a_{\mathbf{J}} \mathbf{x}^{\mathbf{J}}
$$

which is convergent for all elements of the $k$-tuples of $p$-adic integers, and where the right side represents a power series in $k$ variables with $\mathbf{J}$ running through the $k$ tuples of nonnegative integers. In view of the non-archimedean nature of the norm, the convergence of $(6)$ is equivalent to the condition

$$
\lim _{|\mathbf{J}| \rightarrow \infty}\left|a_{\mathbf{J}}\right|_{p}\left|\mathbf{x}^{\mathbf{J}}\right|_{p}=0
$$

PROPOSITION 2. Let $f(\mathbf{x}) \in \mathbb{Z}_{p}\left[\left[x_{1}, \ldots, x_{k}\right]\right]$ be a formal power series in $x_{1}, \ldots, x_{k}$ with $p$-adic integer coefficients, and let

$$
\mathcal{D}=\left\{\left.\mathbf{x} \in \mathbb{C}_{p}^{k}\left|\|\mathbf{x}\|_{p}<1,\|\mathbf{x}\|_{p}=\max _{1 \leq i \leq k}\right| x_{i}\right|_{p}\right\}
$$

be the open polydisc of radius 1 . Then $f(\mathbf{x})$ converges in $\mathcal{D}$.

Proof: Set $\mathbf{x} \in \mathcal{D}$. If $f(\mathbf{x})=\sum_{\mathbf{J}} a_{\mathbf{J}} \mathbf{x}^{\mathbf{J}}, a_{\mathbf{J}} \in \mathbb{Z}_{p}$, where $\mathbf{J}$ runs through the $k$-tuples of nonnegative integers. Then since $\|\mathbf{x}\|_{p}<1$ and $\left|a_{\mathbf{J}}\right|_{p} \leq 1$ for all $\mathbf{J}$, we have $\lim _{|\mathbf{J}| \rightarrow \infty}\left|a_{\mathbf{J}} \mathbf{x}^{\mathbf{J}}\right|_{p}=0$. This completes the proof.

Let $f$ be locally holomorphic on a subset of $\mathbb{C}_{p}^{k}$. By Definition 1 we set

$$
\mu_{0}\left(\mathbf{a}+\mathbf{d} p^{\mathbf{N}} \mathbb{Z}_{p}\right):=\mu_{0}\left(a_{1}+d_{1} p^{N_{1}} \mathbb{Z}_{p}\right) \ldots \mu_{0}\left(a_{k}+d_{k} p^{N_{k}} \mathbb{Z}_{p}\right) .
$$

We also set

$$
S\left(N_{1}, \ldots, N_{k} ; \mathrm{d}\right)=\sum_{x_{1}=0}^{d_{1} p^{N_{1}}-1} \cdots \sum_{x_{k}=0}^{d_{k} p^{N_{k}-1}} f(\mathbf{x}) \mu_{0}\left(\mathbf{x}+\mathbf{d} p^{N} \mathbb{Z}_{p}\right)
$$


From (6) and (8), we can write

$$
\begin{aligned}
S\left(N_{1}, \ldots, N_{k} ; \mathbf{d}\right) & =\sum_{x_{1}=0}^{d_{1} p^{N_{1}}-1} \ldots \sum_{x_{k}=0}^{d_{k} p^{N_{k}-1}}\left(\sum_{\mathbf{J}} a_{\mathbf{J}} \mathbf{x}^{\mathbf{J}}\right) \frac{1}{d_{1} \ldots d_{k} p^{N_{1}} \ldots p^{N_{k}}} \\
& =\sum_{\mathbf{J}} a_{\mathbf{J}}\left(\sum_{x_{1}=0}^{d_{1} p^{N_{1}}-1} \cdots \sum_{x_{k}=0}^{d_{k} p^{N_{k}-1}} \mathbf{x}^{\mathbf{J}} \frac{1}{d_{1} \ldots d_{k} p^{N_{1}} \ldots p^{N_{k}}}\right)
\end{aligned}
$$

and hence

$$
\lim _{\left\{N_{\mathbf{i}}\right\} \rightarrow \infty} S\left(N_{1}, \ldots, N_{k} ; \mathbf{d}\right)=\sum_{\mathbf{J}} a_{\mathbf{J}} B_{\mathbf{J}}
$$

since $B_{n}=\lim _{N \rightarrow \infty} 1 /\left(d p^{N}\right) \sum_{x=0}^{d p^{N}-1} x^{n}$ and each limit is uniform with respect to the remaining variables. Therefore

$$
L=\lim _{\left\{N_{i}\right\} \rightarrow \infty} S\left(N_{1}, \ldots, N_{k} ; \mathbf{d}\right) \text { exists. }
$$

Therefore we have the following definition of a multidimensional Volkenborn integral of a locally holomorphic function.

Definition 3: Let $\mathcal{O}$ be an open set in $\mathbb{C}_{p}^{k}$ with $\mathbf{X} \subset \mathcal{O}$, and let $f: \mathcal{O} \rightarrow \mathcal{B}$ be locally holomorphic, where $\mathcal{B}$ is a Banach space over $\mathbb{C}_{p}$. The multidimensional Volkenborn integral of $f$ is defined by

$$
\begin{aligned}
\int_{\mathbf{X}} f(\mathbf{x}) d \mu_{0}(\mathbf{x}) & :=\int_{X_{d_{1}}} \ldots \int_{X_{d_{k}}} f\left(x_{1}, \ldots, x_{n}\right) d \mu_{0}\left(x_{1}\right) \ldots d \mu_{0}\left(x_{k}\right) \\
& =\lim _{\left\{N_{i}\right\} \rightarrow \infty} \sum_{a_{1}=0}^{d_{1} p^{N_{1}}-1} \cdots \sum_{a_{k}=0}^{d_{k} p^{N_{k}-1}} f(\mathbf{a}) \mu_{0}\left(\mathbf{a}+\mathrm{d} p^{N^{N}} \mathbb{Z}_{p}\right) \\
& =\lim _{\left\{N_{i}\right\} \rightarrow \infty} \sum_{a_{1}=0}^{d_{1} p^{N_{1}}-1} \cdots \sum_{a_{k}=0}^{d_{k} p^{N_{k}-1}} f(\mathbf{a}) \frac{1}{d_{1} \ldots d_{k} p^{N_{1}} \ldots p^{N_{k}}}
\end{aligned}
$$

where $\mathbf{a}=\left(a_{1}, \ldots, a_{k}\right)$ and $\mathbf{d}=\left(d_{1}, \ldots, d_{k}\right) \in \mathbb{N}^{k}$.

For the clarity, in the rest of this paper, we shall denote by 1 the point $(1, \ldots, 1)$.

If we substitute 1 and $N \mathbf{1}$ for $\mathbf{d}$ and $\mathbf{N}$, respectively, then Definition 3 with $f(\mathbf{x})=$ $\left(x_{1}+\cdots+x_{k}\right)^{n}$ suggests a $p$-adic analogue of the higher order Bernoulli number $B_{n}^{(k)}$, which is defined by the generating function $\left(t /\left(e^{t}-1\right)\right)^{k}$.

The following proposition is obvious 
Proposition 3. Let $f$ be a locally holomorphic function on $\mathbb{Z}_{p}^{k}, f(\mathbf{x})$ $=\sum_{\mathbf{J}} a_{\mathbf{J}} \mathbf{x}^{\mathbf{J}} \quad\left(\mathbf{x} \in \mathbb{Z}_{p}^{k}\right)$, and let $\mathbf{J}$ et cetera, be as above. Then we have

$$
\int_{\mathbb{Z}_{p}^{k}}\left(\sum_{\mathbf{J}} a_{\mathbf{J}} \mathbf{x}^{\mathbf{J}}\right) d \mu_{0}(\mathbf{x})=\sum_{\mathbf{J}} a_{\mathbf{J}} \int_{\mathbb{Z}_{p}^{k}} \mathbf{x}^{\mathbf{J}} d \mu_{0}(\mathbf{x})
$$

Now, we consider the function defined on $\mathbb{Z}_{p}^{k}$ by

$$
\mathbf{x} \mapsto|\mathbf{x}|:=x_{1}+\cdots+x_{k}
$$

We shall prove the following generalisation of Proposition 1.

Theorem 4. For $\mathbf{x} \in \mathbb{Z}_{p}^{k}$ let $|\mathbf{x}|^{n}=\left(x_{1}+\cdots+x_{k}\right)^{n}$, where $k \in \mathbb{N}$ and $n \geq 0$. Then

1. $B_{n}^{(k)}=\int_{\mathbb{Z}_{p}^{k}}|\mathbf{x}|^{n} d \mu_{0}(\mathbf{x})$.

2. $B_{n}^{(k)}(x)=\int_{\mathbb{Z}_{p}^{k}}(x+|\mathbf{x}|)^{n} d \mu_{0}(\mathbf{x})$. In particular, $B_{n}^{(k)}(x)=\sum_{i=0}^{n} B_{i}^{(k)}\left(\begin{array}{l}n \\ i\end{array}\right) x^{n-i}$

Proof: Suppose $|t|_{p} \leqslant p^{1 /(1-p)}, t \in \mathbb{C}_{p}$ and $t \neq 0$. First we must show that

$$
\int_{\mathbb{Z}_{p}^{k}} e^{|\mathbf{x}| t} d \mu_{0}(\mathbf{x})=\lim _{N \rightarrow \infty} \frac{1}{p^{k N}} \sum_{x_{1}=0}^{p^{N}-1} \ldots \sum_{x_{k}=0}^{p^{N}-1} e^{\left(x_{1}+\cdots+x_{k}\right) t}=\left(\frac{t}{e^{t}-1}\right)^{k}
$$

The case $k=1$ is familiar from Proposition 1 . For the $k$-dimensional case, by (8), write

$$
\int_{\mathbb{Z}_{p}^{k}} e^{|\mathbf{x}| t} d \mu_{0}(\mathbf{x})=\int_{\mathbb{Z}_{p}} e^{x_{1} t} d \mu_{0}\left(x_{1}\right) \ldots \int_{\mathbb{Z}_{p}} e^{x_{k} t} d \mu_{0}\left(x_{k}\right)
$$

and apply the one-dimensional results. This gives the desired result.

Since we also write $e^{|\mathbf{x}| t}=\sum_{n=0}^{\infty}\left(|\mathbf{x}|^{n} t^{n}\right) /(n !)$, applying term-by-term integration we can readily show that

$$
\sum_{n=0}^{\infty}\left(\int_{\mathbb{Z}_{\boldsymbol{p}}^{k}}|\mathbf{x}|^{n} d \mu_{0}(\mathbf{x})\right) \frac{t^{n}}{n !}=\sum_{n=0}^{\infty} B_{n}^{(k)} \frac{t^{n}}{n !}
$$

This proves the first part. The second part follows in the same fashion.

REMARK. The higher order Bernoulli numbers are a special case of the generalised higher order Bernoulli numbers $B_{n, \chi}^{(k)}$ belong to the Dirichlet character with conductor. It is obvious how to obtain the formulas of Theorem 4 for the generalised higher order Bernoulli numbers $B_{n, \chi}^{(k)}$. 
Corollary 5. For $k \in \mathbb{N}$ and $n \geq 0$ we have

$$
B_{n}^{(k)}=\sum_{i_{1}+\cdots+i_{k}=n}\left(\begin{array}{c}
n \\
i_{1}, \ldots, i_{k}
\end{array}\right) B_{i_{1}} \ldots B_{i_{k}}
$$

where the sum is taken over all nonnegative integers $i_{1}, \ldots, i_{k}$ such that $n=i_{1}+\cdots+i_{k}$, and where $\left(\begin{array}{c}n \\ i_{1}, \ldots, i_{k}\end{array}\right)=(n !) /\left(i_{1} ! \ldots i_{k} !\right)$ is the multinomial coefficient.

Proof: From Theorem 4 we have

$$
\begin{aligned}
B_{n}^{(k)} & =\lim _{N \rightarrow \infty} \frac{1}{p^{k N}} \sum_{x_{1}=0}^{p^{N}-1} \ldots \sum_{x_{k}=0}^{p^{N}-1}\left(x_{1}+\cdots+x_{k}\right)^{n} \\
& =\sum_{i_{1}+\cdots+i_{k}=n}\left(\begin{array}{c}
n \\
i_{1}, \ldots, i_{k}
\end{array}\right) \lim _{N \rightarrow \infty} \frac{1}{p^{k N}} \sum_{x_{1}=0}^{p^{N}-1} \ldots \sum_{x_{k}=0}^{p^{N}-1} x_{1}^{i_{1}} \ldots x_{k}^{i_{k}} .
\end{aligned}
$$

We therefore obtain the corollary.

EXAMPLE 1. In fact $B_{0}=1, B_{1}=-(1 / 2), B_{2}=1 / 6, B_{3}=0$ and $0 !=1$, we have

1. For $n=1, B_{1}^{(k)}=\sum_{i_{1}+\cdots+i_{k}=1}\left(\begin{array}{c}1 \\ i_{1}, \ldots, i_{k}\end{array}\right) B_{i_{1}} \ldots B_{i_{k}}=k B_{1}=-(1 / 2) k$.

2. For $n=2$,

$$
\begin{aligned}
B_{2}^{(k)} & =\sum_{i_{1}+\cdots+i_{k}=2}\left(\begin{array}{c}
2 \\
i_{1}, \ldots, i_{k}
\end{array}\right) B_{i_{1}} \ldots B_{i_{k}} \\
& =\frac{k(k-1)}{2}\left(\begin{array}{c}
2 \\
1,1
\end{array}\right) B_{1} B_{1}+k\left(\begin{array}{l}
2 \\
2
\end{array}\right) B_{2}=\frac{k}{12}(3 k-1) .
\end{aligned}
$$

3. For $n=3$,

$$
\begin{aligned}
B_{3}^{(k)} & =\sum_{i_{1}+\cdots+i_{k}=3}\left(\begin{array}{c}
3 \\
i_{1}, \ldots, i_{k}
\end{array}\right) B_{i_{1}} \ldots B_{i_{k}} \\
& =\frac{k(k-1)(k-2)}{3 !}\left(\begin{array}{c}
3 \\
1,1,1
\end{array}\right) B_{1} B_{1} B_{1}+k(k-1)\left(\begin{array}{c}
3 \\
1,2
\end{array}\right) B_{1} B_{2}+k\left(\begin{array}{l}
3 \\
3
\end{array}\right) B_{3} \\
& =-\frac{1}{8} k^{2}(k-1) .
\end{aligned}
$$

EXAMPLE 2. Corollary 5 and the formula [9, p. 145]

$$
B_{n}^{(k+1)}(x)=\left(1-\frac{n}{k}\right) B_{n}^{(k)}(x)+(x-k) \frac{n}{k} B_{n-1}^{(k)}(x)
$$


give rise to more identities. For example, we obtain for $k=1$ trivially $B_{n}^{(1)}=B_{n}$, and

$$
\begin{aligned}
B_{n}^{(2)}= & \sum_{\substack{i_{1}, i_{2} \geq 0 \\
i_{1}+i_{2}=n}}\left(\begin{array}{c}
n \\
i_{1}, i_{2}
\end{array}\right) B_{i_{1}} B_{i_{2}}=\sum_{i=0}^{n}\left(\begin{array}{c}
n \\
i
\end{array}\right) B_{i} B_{n-i}=(1-n) B_{n}-n B_{n-1} \\
B_{n}^{(3)}= & \sum_{\substack{i_{1}, i_{2}, i_{3} \geq 0 \\
i_{1}+i_{2}+i_{3}=n}}\left(\begin{array}{c}
n \\
i_{1}, i_{2}, i_{3}
\end{array}\right) B_{i_{1}} B_{i_{2}} B_{i_{3}} \\
= & \frac{1}{2}(n-1)(n-2) B_{n}+\frac{3}{2} n(n-2) B_{n-1}+n(n-1) B_{n-2} \\
B_{n}^{(4)}= & \sum_{\substack{i_{1}, i_{2}, i_{3}, i_{4} \geq 0 \\
i_{1}+i_{2}+i_{3}+i_{4}=n}}\left(\begin{array}{c}
n \\
i_{1}, i_{2}, i_{3}, i_{4}
\end{array}\right) B_{i_{1}} B_{i_{2}} B_{i_{3}} B_{i_{4}} \\
= & -\frac{1}{6}(n-1)(n-2)(n-3)-n(n-2)(n-3) B_{n-1} \\
& \quad-\frac{11}{6} n(n-1)(n-3) B_{n-2}-n(n-1)(n-2) B_{n-3} .
\end{aligned}
$$

Similarly, one can also evaluate the sum

$$
B_{n}^{(k)}=\sum_{\substack{i_{1}, \ldots, i_{k} \geq 0 \\
i_{1}+\cdots+i_{k}=n}}\left(\begin{array}{c}
n \\
i_{1}, \ldots, i_{k}
\end{array}\right) B_{i_{1}} \ldots B_{i_{k}}
$$

However the formula will be a little bit lengthy.

Corollary 6. Let $\alpha=\alpha_{1}+\cdots+\alpha_{k}$ with $\alpha_{1}, \ldots, \alpha_{k} \in \mathbb{Z}_{p}$, and let $k \in \mathbb{N}$ and $n \geq 0$. Then

$$
B_{n}^{(k)}\left(\alpha_{1}+\cdots+\alpha_{k}\right)=\sum_{i_{1}+\cdots+i_{k}=n}\left(\begin{array}{c}
n \\
i_{1}, \ldots, i_{k}
\end{array}\right) B_{i_{1}}\left(\alpha_{1}\right) \ldots B_{i_{k}}\left(\alpha_{k}\right),
$$

where the sum is taken over all nonnegative integers $i_{1}, \ldots, i_{k}$ such that $n=i_{1}+\cdots+i_{k}$, and where $\left(\begin{array}{c}n \\ i_{1}, \ldots, i_{k}\end{array}\right)=(n !) /\left(i_{1} ! \ldots i_{k} !\right)$ is the multinomial coefficient.

PROOF: This follows as in the proof of Corollary 5.

ExAmPLE 3. From Equation (12) and Corollary 6, we have

$$
\begin{aligned}
\text { 1. } & k=1: B_{n}^{(1)}(1)=B_{n}, B_{n}^{(1)}(1 / 2)=-\left(1-2^{1-n}\right) B_{n} \\
\text { 2. } & k=2: \text { For } \alpha=\alpha_{1}+\alpha_{2} \\
B_{n}^{(2)}(\alpha)= & \sum_{\substack{i_{1}, i_{2} \geq 0 \\
i_{1}+i_{2}=n}}\left(\begin{array}{c}
n \\
i_{1}, i_{2}
\end{array}\right) B_{i_{1}}\left(\alpha_{1}\right) B_{i_{2}}\left(\alpha_{2}\right)=(1-n) B_{n}(\alpha)+n(\alpha-1) B_{n-1}(\alpha) .
\end{aligned}
$$

3. $k=3$ : For $\alpha=\alpha_{1}+\alpha_{2}+\alpha_{3}$, 


$$
\begin{aligned}
B_{n}^{(3)}(\alpha)= & \sum_{\substack{i_{1}, i_{2}, i_{3} \geq 0 \\
i_{1}+i_{2}+i_{3}=n}}\left(\begin{array}{c}
n \\
i_{1}, i_{2}, i_{3}
\end{array}\right) B_{i_{1}}\left(\alpha_{1}\right) B_{i_{2}}\left(\alpha_{2}\right) B_{i_{3}}\left(\alpha_{3}\right) \\
= & \frac{1}{2}(n-1)(n-2) B_{n}(\alpha)-\frac{1}{2} n(n-2)(2 \alpha-3) B_{n-1}(\alpha) \\
& +\frac{1}{2} n(n-1)(\alpha-1)(\alpha-2) B_{n-2}(\alpha)
\end{aligned}
$$

4. The sums of products of Bernoulli polynomials in Corollary 6 can also be evaluated by a slightly longer calculation.

LEMmA 7 . For any integer $i_{1}, \ldots, i_{k} \geq 0$ with $n=i_{1}+\cdots+i_{k}$ we have

$$
\left|\left(\begin{array}{c}
n \\
i_{1}, \ldots, i_{k}
\end{array}\right)\right|_{p} \leq 1, \quad \text { that is }\left(\begin{array}{c}
n \\
i_{1}, \ldots, i_{k}
\end{array}\right) \in \mathbb{Z}_{p}
$$

Proof: Put $n=i_{1}+\cdots+i_{k}$. We must show that

$$
\operatorname{ord}_{p}\left(i_{1} ! \ldots i_{k} !\right) \leq \operatorname{ord}(n !)
$$

since $\left(\begin{array}{c}n \\ i_{1}, \ldots, i_{k}\end{array}\right)=(n !) /\left(i_{1} ! \ldots i_{k} !\right)$. Using [] to denote the greatest integer function, we have

$$
\begin{aligned}
\operatorname{ord}_{p}\left(i_{1} ! \ldots i_{k} !\right) & =\sum_{l=1}^{\infty}\left(\left[\frac{i_{1}}{p^{l}}\right]+\cdots+\left[\frac{i_{k}}{p^{l}}\right]\right) \leq \sum_{l=1}^{\infty}\left[\frac{i_{1}+\cdots+i_{k}}{p^{l}}\right]=\sum_{l=1}^{\infty}\left[\frac{n}{p^{l}}\right] \\
& =\operatorname{ord}_{p}(n !)
\end{aligned}
$$

because $[x]+[y] \leq[x+y]$ for all real $x, y$. This completes the proof.

A proof of the following lemma can be found in [7, Chapter 2].

LEMMA 8. If $n$ is a positive integer with $p-1 \nmid n$, then $\left|B_{n} / n\right|_{p} \leq 1$.

To state our results we shall require some notation. Let $\Lambda_{k}(n)$ denote the number of sets of nonnegative integer solutions $\left(i_{1}, \ldots, i_{k}\right)$ to the equation

$$
i_{1}+\cdots+i_{k}=n \quad \text { for some } p-1 \mid i_{l}, l=1, \ldots, k .
$$

THEOREM 9. For all $n>0$ we have

$$
B_{n}^{(k)}-\sum_{\left(i_{1}, \ldots, i_{k}\right) \in \Lambda_{k}(n)}\left(\begin{array}{c}
n \\
i_{1}, \ldots, i_{k}
\end{array}\right) B_{i_{1}} \ldots B_{i_{k}} \in \mathbb{Z}_{p}
$$

Proof: By Corollary 5, we have

$$
B_{n}^{(k)}=\sum_{i_{1}+\cdots+i_{k}=n}\left(\begin{array}{c}
n \\
i_{1}, \ldots, i_{k}
\end{array}\right) B_{i_{1}} \ldots B_{i_{k}} .
$$




\section{From Lemma 7}

$$
\left|B_{n}^{(k)}-\sum_{\left(i_{1}, \ldots, i_{k}\right) \in \Lambda_{k}(n)}\left(\begin{array}{c}
n \\
i_{1}, \ldots, i_{k}
\end{array}\right) B_{i_{1}} \ldots B_{i_{k}}\right| \leq\left.\max _{p}\left|B_{\substack{i_{1}+\cdots+i_{k}=n \\
p-1 \nmid i_{1}, \ldots, p-1 \nmid i_{k}}}\right| B_{i_{1}} \ldots B_{i_{k}}\right|_{p} .
$$

Applying Lemma 8 to the right side of this inequality, one finds

$$
\max _{\substack{i_{1}+\cdots+i_{k}=n \\ p-1 \nmid i_{1}, \ldots, p-1 \nmid i_{k}}}\left|B_{i_{1}} \ldots B_{i_{k}}\right|_{p} \leq \max _{\substack{i_{1}+\cdots+i_{k}=n \\ p-1 \nmid i_{1}, \ldots, p-1 \nmid i_{k}}}\left|i_{1} \ldots i_{k}\right|_{p} .
$$

Using the result that $i_{1} \ldots i_{k} \in \mathbb{Z}_{p}$ for all $i_{1}, \ldots, i_{k} \geq 0$, we have

$$
\max _{\substack{i_{1}+\cdots+i_{k}=n \\ p-1 \nmid i_{1}, \ldots, p-1 \nmid i_{k}}}\left|i_{1} \ldots i_{k}\right|_{p} \leq 1
$$

which completes the proof for all $n>0$.

Part 1 of the following theorem was first introduced by Mahler [8]. He was interested mainly in functions of one variable, for the purpose of making the similarities and the differences between real and $p$-adic functions more intelligible. Also, in $[8$, Chapter $12]$, he extended some of the function theory of one variable to the case of functions of two variables.

THEOREM 10. (Mahler expansion and base for $C\left(\mathbb{Z}_{p}^{k} \rightarrow \mathbb{C}_{p}\right)$.) Let $C\left(\mathbb{Z}_{p}^{k} \rightarrow \mathbb{C}_{p}\right)$ be the set of all continuous functions $\mathbb{Z}_{p}^{k} \rightarrow \mathbb{C}_{p}$. Let $\mathbf{J}=\left(j_{1}, \ldots, j_{k}\right)$ be multi-index of the $k$-tuples of nonnegative integers, and $|\mathrm{J}|=j_{1}+\cdots+j_{k}$.

1. If $f \in C\left(\mathbb{Z}_{p}^{k} \rightarrow \mathbb{C}_{p}\right)$ has the Mahler expansion

$$
f\left(x_{1}, \ldots, x_{k}\right)=\sum_{\mathbf{J}} a_{\mathbf{J}}\left(\begin{array}{c}
x_{1} \\
j_{1}
\end{array}\right) \ldots\left(\begin{array}{c}
x_{k} \\
j_{k}
\end{array}\right)
$$

where $\mathbf{J}$ runs through the $k$-tuples of nonnegative integers, then

$$
a_{\mathbf{J}}=\sum_{i_{1}=0}^{j_{1}} \ldots \sum_{i_{k}=0}^{j_{k}}(-1)^{|\mathbf{J}|-\left(i_{1}+\cdots+i_{k}\right)}\left(\begin{array}{c}
j_{1} \\
i_{1}
\end{array}\right) \ldots\left(\begin{array}{c}
j_{k} \\
i_{k}
\end{array}\right) f\left(i_{1}, \ldots, i_{k}\right) .
$$

2. The function

$$
\left(x_{1}, \ldots, x_{k}\right) \mapsto\left(\begin{array}{c}
x_{1} \\
j_{1}
\end{array}\right) \ldots\left(\begin{array}{c}
x_{k} \\
j_{k}
\end{array}\right)
$$

forms an orthonormal basis of $C\left(\mathbb{Z}_{p}^{k} \rightarrow \mathbb{C}_{p}\right)$. 
Proof: The set $\left\{\left(\begin{array}{l}x \\ m\end{array}\right)\left(\begin{array}{l}y \\ n\end{array}\right): m, n \geq 0\right.$ and $\left.(x, y) \in \mathbb{Z}_{p}^{2}\right\}$ is an orthonormal basis of $C\left(\mathbb{Z}_{p}^{2} \rightarrow \mathbb{C}_{p}\right)$, topologised by the sup-norm. Similarly, $\left\{\left(\begin{array}{c}x_{1} \\ j_{1}\end{array}\right) \ldots\left(\begin{array}{c}x_{k} \\ j_{k}\end{array}\right)\right\}$ is an orthonormal basis of $C\left(\mathbb{Z}_{p}^{k} \rightarrow \mathbb{C}_{p}\right)$, topologised by the sup-norm (see [11]).

From this we deduce the following example.

EXAMPLE 4. Let $a$ be a $p$-adic number satisfying $|a|<p^{1 /(1-p)}$. Then for $x_{1}, \ldots, x_{k}$ $\in \mathbb{Z}_{p}$ we have
1. $\quad a^{x_{1}+\cdots+x_{k}}=\sum_{j_{k}=0}^{\infty} \ldots \sum_{j_{1}=0}^{\infty}(a-1)^{j_{1}+\cdots+j_{k}}\left(\begin{array}{l}x_{1} \\ j_{1}\end{array}\right) \ldots\left(\begin{array}{c}x_{k} \\ j_{k}\end{array}\right)$.

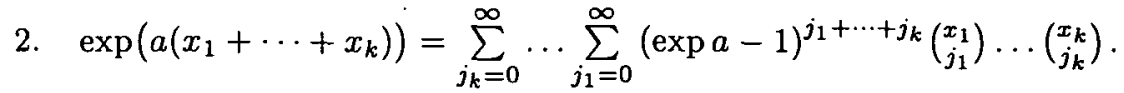

Proof: To see Part 1, the binomial theorem, readily shows that

$$
a^{m_{1}+\cdots+m_{k}}=\sum_{j_{k}=0}^{m_{k}} \ldots \sum_{j_{1}=0}^{m_{1}}(a-1)^{j_{1}+\cdots+j_{k}}\left(\begin{array}{c}
m_{1} \\
j_{1}
\end{array}\right) \cdots\left(\begin{array}{c}
m_{k} \\
j_{k}
\end{array}\right)
$$

for $m_{1}, \ldots, m_{k} \in \mathbb{N}$. Now continuity (see [7, Chapter 2]) proves Part 1.

Since $|a|_{p}<p^{1 /(1-p)}$ and $x_{1}+\cdots+x_{k} \in \mathbb{Z}_{p}$,

$$
\exp \left(a\left(x_{1}+\cdots+x_{k}\right)\right)=(\exp a)^{x_{1}+\cdots+x_{k}}
$$

(see [11]). Hence Part 1 gives Part 2.

EXAMPLE 5. Let $\zeta$ be a root of unity of order $p$, a prime number $p \neq 2$. Then

$$
\sum_{\substack{i_{1}+\cdots+i_{p-1}=l \\
i_{1}, \ldots, i_{p-1} \geq 0}} \zeta^{i_{1}+2 i_{2}+\cdots+(p-1) i_{p-1}}=\left\{\begin{aligned}
1 & \text { if } l \equiv 0(\bmod p), \\
-1 & \text { if } l \neq \equiv(\bmod p), \\
0 & \text { otherwise. }
\end{aligned}\right.
$$

PROOF: Let

$$
f(x)=\sum_{n=0}^{\infty} x^{n}=1+x+\cdots+x^{n}+\cdots
$$

For $\zeta \in \mathbb{C}_{p}$, let $\zeta$ be a primitive $p$-th root of unity. We set $F(x):=\prod_{\alpha=1}^{p-1} f\left(\zeta^{\alpha} x\right)$. It is thus easy to see that

$$
F(x)=\sum_{l=0}^{\infty} a_{l} x^{l}
$$


where

$$
a_{l}=\sum_{\substack{i_{1}+\cdots+i_{p-1}=l \\ i_{1}, \ldots, i_{p-1} \geq 0}} \zeta^{i_{1}+2 i_{2}+\cdots+(p-1) i_{p-1}} .
$$

For $|x|_{p}<1, f(x)$ converges to $1 /(1-x)$. One also sees that

$$
\begin{aligned}
F(x) & =\prod_{\alpha=1}^{p-1} \frac{1}{1-\zeta^{\alpha} x}=\frac{1-x}{\prod_{\alpha=1}^{p}\left(1-\zeta^{\alpha} x\right)}=\frac{1-x}{1-x^{p}} \\
& =(1-x)\left(1+x^{p}+x^{2 p}+\cdots+x^{n p}+\cdots\right) .
\end{aligned}
$$

The result now follows upon equating the coefficients of $x^{l}$ on both sides of the above equation.

COROLLARY 11. Let $f$ et cetera, be as in Theorem 10. Then

$$
\int_{\mathbb{Z}_{p}^{k}} f(\mathbf{x}) d \mu_{0}(\mathbf{x})=\sum_{\mathbf{J}} a_{\mathbf{J}} \frac{(-1)^{|\mathbf{J}|}}{\left(j_{1}+1\right) \ldots\left(j_{k}+1\right)} \quad\left(\mathbf{x} \in \mathbb{Z}_{p}^{k}\right) .
$$

Proof: Note that

$$
\int_{\mathbb{Z}_{p}}\left(\begin{array}{l}
x \\
n
\end{array}\right) d \mu_{0}(x)=\frac{(-1)^{n}}{n+1}
$$

for all $n \geq 0$ (see $[\mathbf{8}, \mathbf{1 0}, \mathbf{1 1}])$. Thus the result follows from Theorem 10 .

\section{REFERENCES}

[1] A. Adelberg, 'Congruences of $p$-adic integer order Bernoulli numbers', J. Number Theory 59 (1996), 374-388.

[2] L. Carlitz, 'Some theorems on Bernoulli numbers of higher order', Pacific J. Math. 2 (1952), 127-139.

[3] J. Diamond, 'The $p$-adic log gamma function and $p$-adic Euler constants', Trans. Amer. Math. Soc. 233 (1977), 321-337.

[4] K. Dilcher, 'Sums of products of Bernoulli numbers', J. Number Theory 60 (1996), 23-41.

[5] F. T. Howard, 'Congruences and recurrences for Bernoulli numbers of higher order', $F i$ bonacci Quart. 32 (1994), 316-328.

[6] N. Koblitz, p-Adic Analysis: a Short Course on Recent Work, London Mathematical Society Lecture Notes 46 (Cambridge University Press, Cambridge, New York, 1980).

[7] N. Koblitz. p-adic Numbers, p-adic Analysis and Zeta-Functions, Graduate Texts in Mathematics 58 (Springer-Verlag, Berlin, Heidelberg, New York, 1984).

[8] K. Mahler, p-adic numbers and their functions, Cambridge tracts in mathematics 76 (Cambridge University Press, Cambridge, New York, 1981).

[9] N. E. Nörlund, Vorlesungen über Differenzenrechnung (Springer, Berlin, 1924). 
[10] A. M. Robert. A Course in p-adic Analysis (Springer-Verlag, Berlin, Heidelberg, New York, 2000).

[11] W. H. Schikhof, Ultrametric Calculus, An introduction to $p$-adic analysis, Cambridge Studies in Adv. Math. 4 (Cambridge University Press, Cambridge, New York, 1984).

[12] L. C. Washington, Introduction to Cyclotomic Fields, Graduate Texts in Mathematics 83 (Springer-Verlag, Berlin, Heidelberg, New York, 1997).

[13] C. F. Woodcock, 'An invariant $p$-adic integral on $\mathbb{Z}_{p}$ ', J. London Math. Soc. 8 (1974), 731-734.

[14] P. T. Young, 'Congruences for Bernoulli, Euler, and Stirling numbers', J. Number Theory 78 (1999), 204-227.

Department of Mathematics

Kyungnam University

Masan 631-701

South Korea

e-mail: mskim@mail.kyungnam.ac.kr
Department of Mathematics

Kyungnam University

Masan 631-701

South Korea

e-mail: sonjin@hanma.kyungnam.ac.kr 